\title{
Scheduling of measles vaccination in low-income countries: projections of a dynamic model
}

\author{
C.T. Bauch ${ }^{1} *$, E. Szusz ${ }^{1}$, L.P. Garrison ${ }^{2}$ \\ 1: Dept. of Mathematics and Statistics, Univ. Guelph, Guelph, ON N1G 2W1, Canada \\ 2: Dept. of Pharmacy, Univ. Washington, Box 357630 , Seattle, WA 98195-7630, USA
}

*Author for correspondence

Email: cbauch@uoguelph.ca

Phone: 519-824-4120 ext 53079

Fax: 519-837-0221

Submitted to Vaccine, January 26, 2008

First revision submitted March 9, 2009

Second revision submitted April 3, 2009 


\begin{abstract}
Large-scale vaccination campaigns (SIAs) and improved routine immunization (RI) have greatly reduced measles incidence in low-income countries. However, the interval between SIAs required to maintain these gains over the long term is not clear. We developed a dynamic model of measles transmission to assess measles vaccination strategies in Cambodia, Ghana, India, Morocco, Nigeria, and Uganda. We projected measles cases from 2008-2050 under (a) holding SIAs every 2, 4, 6, or 8 years (b) improvements in first dose routine measles vaccine (MCV1) coverage of $0 \%, 1 \%, 3 \%$ annually, and (c) introducing MCV2 once MCV1 coverage reaches 70\%, 80\%, 90\%. If MCV1 continues improving, then India and Nigeria could hold SIAs every 4 years without significant probability of large outbreaks, and the other countries every 6-8 years. If RI remains stagnant, India and Nigeria should hold SIAs every 2 years, and the other countries every 4-6 years.
\end{abstract}

Keywords: compartmental model, measles vaccine, supplementary immunization activities, dynamic model, global health, second opportunity 


\section{Introduction}

In the 1990s, endemic measles was dramatically reduced and — according to many— completely eliminated in the Americas through large-scale supplemental immunization activities (SIAs) and efforts to strengthen routine immunization (RI) programs by improving coverage of the first scheduled dose of measles-containing vaccine (MCV1) and by introducing a second scheduled dose (MCV2) (1). Subsequently, these "second opportunity" strategies were extended to 45 priority countries, mostly in Africa and Asia (1). As a result, the estimated number of worldwide measles-related deaths declined from 873,000 in 1999 to 345,000 in 2005 (1). However, measles disease burden remains high in absolute terms, and many of these countries only recently held their first SIA and are far from introducing $\operatorname{MCV} 2(1,2)$. Therefore, decision-makers continue to face challenges in determining the optimal interval between SIAs and the optimal criteria for introducing MCV2 in the priority countries. At the same time, new measles vaccine technologies are in development. These include DNA primers that make conventional measles vaccine more effective in infants (3). Other technologies simplify administration and waste disposal by administering vaccine via aerosolized droplets (4), dry powder formulations (5), and needle-free syringes (6). Decision-makers therefore also face challenges in determining how to invest in these new technologies, particularly given the rapidly changing landscape of global measles disease burden.

To meet these challenges, credible projections of future measles cases under various possible vaccination strategies are needed. Such projections can be obtained using 
dynamic disease transmission models (7). Dynamic models incorporate transmission mechanisms and can thereby capture herd immunity effects, whereby vaccination also protects unvaccinated individuals by reducing disease transmission in the population and thus reducing the force of infection (the rate at which a susceptible person is infected) (8). As a result, an infectious disease can be eliminated in a population with an imperfectly efficacious vaccine, and without vaccinating everyone. Herd immunity effects become particularly pronounced at higher coverage levels, near the elimination threshold in vaccine coverage (8). Hence, dynamic models are excellent choices for projecting the impact of vaccination programs when coverage levels are high, as might occur under successful measles SIA and RI efforts. Dynamic models are referred to as dynamic because they can capture how the force of infection evolves over time due to factors such as the introduction of vaccination. By comparison, widely-used static models (e.g. cohort models) assume a fixed, unchanging force of infection and do not capture herd immunity (9). Therefore, they incorrectly predict that disease elimination can only occur when a perfectly efficacious vaccine is given to everyone in a population, which contradicts the local elimination of measles that has been observed in many countries immediately following SIA efforts.

A dynamic model of particular relevance for measles is the age-structured SEIR compartmental model. This model and variations thereof have long been applied for assessing vaccination programs for pediatric infectious diseases, and has been validated against pre- and post-vaccination age-stratified case reports and seroprevalence surveys for measles $(8,10-12)$. Previous analyses have tailored measles models to settings where 
birth rates and transmission rates are high $(13,14)$ as well as campaign settings $(15,16)$. The SEIR model has also been applied in very policy-specific settings, such as in New Zealand where an age-structured measles model was used by the Ministry of Health to predict an epidemic of measles and to design optimal vaccination schedules for that country (17). The SEIR model has since been developed into metapopulation models of measles transmission, such as have been used recently to understand the spatio-temporal dynamics of measles outbreaks in western Africa (18).

The objective of this study was to develop a simplified compartmental model of measles transmission and vaccination in low-income settings. The model was developed for use in a pilot project that will assess the cost-effectiveness of potential innovations in measles vaccination technologies in low-income countries. Here, we demonstrate the stand-alone utility of the model for assessing the effectiveness of second-opportunity strategies with the current vaccine. A model with relatively simple structure was developed because there are often not sufficient data for many low-income countries to populate the parameters of more complex models. As a result, we did not include stochastic, spatial, seasonal or other effects that are relevant in certain contexts $(8,12,18,19)$. However, because of its limited data requirements, the model can be easily adapted to any given country. The present model requires country-specific vaccine coverage, vital statistics, and (optionally) measles case fatality rate. Most other parameters are specific to lowincome countries generally. 
Accordingly we developed country-specific versions of the model for six countries, chosen on the basis of their relatively high annual number of cases (India, Nigeria), the availability of vaccine cost data that will be used in the pilot project on cost-effectiveness that will use this model (Cambodia, Ghana, Morocco), or because they provide illustrative examples of cases where data availability is quite limited (India, Uganda). These countries also represent a wide range of measles disease burden and vaccine coverage. To illustrate the utility and specific policy implications of the model, we explored projected cases in these countries under various possible scenarios for (1) interval between SIAs, (2) criteria for introducing MCV2, and (3) rate of improvement of MCV1 coverage. We also compared the projections of the compartmental model to those of the corresponding static model.

\section{Methods}

\subsection{Model structure}

We developed an age-structured MSEIRV compartmental model, whereby individuals are allocated into one of a number of mutually exclusive categories based on their epidemiological status and age. Epidemiological categories were: maternally immune (naturally-derived), maternally immune (vaccine-derived), susceptible, exposed, infectious, recovered, and vaccinated. We distinguished between vaccine- and naturallyderived maternal immunity because they wane at different rates $(20,21)$. We define naturally-derived maternal immunity as maternal immunity conferred to a child by a 
mother who was exposed to measles infection in the past (and may or may not have been vaccinated), and we define vaccine-derived maternal immunity as maternal immunity conferred to a child by a mother who was vaccinated against measles but never infected. Age classes were: $<1$ month old, 1 month old, 2 months old, ..., 59 months old, 5 years old, 6 years old, ..., 9 years old, 10-14 years old, 15-19 years old, .., 75-79 years old. Fine age stratification in the younger age classes can allow us to study the impact of measles innovations such as the DNA vaccine under the associated pilot project (DNA priming may make conventional vaccine more effective below 9 months of age (3)). Although the model structure allows for a large upper limit on ages, we note that the actual sizes of older age compartments are small since the model is parameterized with country-specific historical and projected demographic data (22). We do not include seasonal forcing in the baseline model since there are little data that can be use to estimate country-specific estimates of seasonal forcing amplitudes. However, the impact of seasonal forcing on model outcomes is explored in the Results. We also assume that vaccine-derived immunity wanes at some specified rate $(23,24)$. Figure 1 presents a schematic diagram of the model. Model equations appear in Supplementary Appendix 1.

The total number of susceptible (resp. exposed, infectious, etc.) individuals in age class $i$ at a given time is denoted $S_{\mathrm{i}}\left(\operatorname{resp} . E_{\mathrm{i}}, I_{\mathrm{i}}, e t c\right)$. Individuals are born at rate $b(t)$ per year, where the birth rate can depend on the year $t$ according to historical demographic data and future projections. Individuals are born with naturally derived maternal immunity, vaccine-derived maternal immunity, or are susceptible. The relative proportion of individuals born into each of these three categories at a given time depends upon the 
number of women of childbearing years in the $R_{\mathrm{i}}, V_{\mathrm{i}}$, or $S_{\mathrm{i}}$ compartments at that time, respectively. Individuals in age class $i$ with naturally- (resp. vaccine-) derived maternal immunity lose it at rate $\rho_{i}^{N}$ (resp. $\rho_{i}^{V}$ ), becoming fully susceptible. Susceptible

individuals in age class $i$ become infected at rate $\lambda_{i}=\sum_{j=1}^{80} \beta_{i j} I_{j} / N_{j}$, where $N_{\mathrm{j}}$ is the total number of individuals of age class $j$ and $\beta_{\mathrm{ij}}$ is the rate at which an infectious person of age class $j$ transmits to a susceptible person of age class $i$. Newly infected individuals remain "exposed" for $1 / \sigma$ days on average and then enter the "infectious" class, where they remain for $1 / \gamma$ days on average. After this, a proportion $d_{i}^{M}$ of individuals die from measles-related complications, while the remainder acquired lifelong immunity and enter the $R_{\mathrm{i}}$ class. A proportion $\varepsilon_{\mathrm{i}}$ of vaccinees enter the $V_{\mathrm{i}}$ class $\left(\varepsilon_{\mathrm{i}}\right.$ is the vaccine effectiveness) while $1-\varepsilon_{\mathrm{i}}$ remain susceptible. Individuals in the vaccinated class lose their immunity at rate $w$, thereby becoming fully susceptible again. Each month, individuals of age class $i$ move to age class $i+1$ (i.e., they age by one month), except for a proportion $d_{\mathrm{i}}(t)$ who die due to causes other than measles.

\subsection{Review of epidemiologic data for low-income countries}

Measles incidence patterns can vary significantly across populations according to population density $(7,8)$, or behavioural factors $(13,25)$. However, measles epidemiology tends to exhibit certain broad features in low-income countries, as compared to high-income countries (13). For instance, the mean age at infection in unvaccinated populations in low-income countries is 1-3 years, which is much lower than the 4-6 years observed before mass vaccination in high-income countries in the twentieth 
century $(8,13,25,26)$. Supplementary Appendix 2 summarizes eight studies reporting age-stratified attack rates in Indian and African populations from the 1970s and 1980s, before vaccination was widespread. The summary shows that the measles attack rate peaks in $<1,1$, or 2-year-olds. Supplementary Appendix 2 also summarizes the percent of children exhibiting measles antibodies by age 5 according to eight seroprevalence surveys from Indian and African populations in the 1970s and 1980s. The average percent infected by age 5 was $78 \%$, with most studies falling between $70 \%$ and $90 \%$. The force of infection implied by these seroprevalence studies is consistent with a mean age of infection below 3 years of age, though we note that force of infection estimates based on seroprevalence surveys can be biased downward if they do not account for diseaserelated mortality, which is significant for measles in low-income countries $(13,27)$.

Case reporting time series submitted to the World Health Organization (WHO) appear in Figure 2 for the six countries (28). These data sources vary considerably in quality and are often unreliable. However, clear patterns emerge for some countries. The Nigerian data reveal a typical measles epidemic pattern from 1990 to 2005: measles epidemic activity peaks every 2 years, and sometimes every 3 years (auto-correlation=0.39 at lag 1). The presence of a 2-year cycle in aggregated country-level data suggests that Nigerian population centres may have experienced synchronized outbreaks after 1990, perhaps even being driven by Lagos. Similar phenomena have been observed in highincome countries with large capital populations (19). The correlation coefficient $C$ between the case reporting and WHO-UNICEF estimated MCV1 coverage time series for the same time period can be computed for Cambodia $(C=-0.69)$, Ghana $(C=-0.70)$, India 
$(C=-0.79)$, Morocco $(C=-0.87)$, Nigeria $(C=-0.61)$, and Uganda $(C=-0.01) . C$ is generally large and negative, suggesting that the trends observed in the case reports substantially reflect true changes in disease incidence due to changing vaccine coverage. The sole exception is Uganda, for which the quality of the case reporting time series appears to be very poor (note the missing data points and the implausibly low numbers of case reports in the late 1980s).

\subsection{Model parameterization}

The baseline parameter values with corresponding data sources appear in Table 1. All parameters were set according to estimates in the literature $(2,22,24,28-40)$, except the rates of waning of maternal immunity and the age-stratified force of infection, which were fitted to data $(20,21,41,42)$. To parameterize rates of waning maternal immunity, we fitted data from the literature to a mathematical model (see Online Appendix 1 for details). Subsequently, to parameterize the age-stratified force of infection, the output from the dynamic model in the absence of vaccination was closely matched to estimates of basic epidemiological measures as summarized in the foregoing section, yielding (1) a mean at age infection of $A \approx 2.3$ years, (2) highest attack rates in 1-year-olds, followed closely by those under 1 year of age, and (3) $86 \%$ of 5 -year-olds with a history of natural measles infection. We note that because most measles infection occurs in childhood, it is difficult to estimate force of infection above 15 years of age. Therefore, we made our baseline values in these age classes consistent with estimates from populations where higher quality data sets are available for older ages $(8,13)$. A contact surface, rather than 
a WAIFW matrix, was used to compute transmission rates from the age-stratified force of infection (see Online Appendix 1 for details) (43). A contact surface requires fewer free parameters than the corresponding WAIFW matrix for finely stratified age classes. Immigration of exposed individuals from other populations was assumed to occur at a constant rate of $N \times 10^{-6}$ per year (44), where $N=$ population size, in order to provide a floor for simulated incidence. To quantify waning of vaccine-derived immunity, we used studies on waning of antibodies and did not consider waning of cellular immunity.

\subsection{Uncertainty analysis}

We carried out a probabilistic uncertainty analysis with respect to major epidemiological parameters. We defined a triangular distribution about the baseline parameter values for duration of latency $(-20 \%,+20 \%)$, duration of infectiousness $(-20 \%,+20 \%)$, vaccine efficacy at 9 months $(-5 \%,+5 \%)$, vaccine efficacy at 12 months $(-5 \%,+5 \%)$, rate of waning of vaccine-derived immunity $(-50 \%,+50 \%)$, and force of infection $(-20 \%,+20 \%)$ (lower and upper limits are given parenthetically). Narrow ranges for sensitivity analysis were used for parameter values that are known relatively well (e.g. clinical vaccine efficacy) whereas wider ranges were used for parameters that are known with least accuracy (e.g. rate of waning vaccine-derived immunity). We ran 100 realizations for each scenario, sampling values from the triangular distributions for each parameter every time. We ran 100 realizations because the standard deviation appeared to converge after this number of simulations. 


\subsection{Model validation}

The dynamic model was validated by comparing its output to independent estimates of the basic reproduction number $R_{0}$ and to case reporting time series. $R_{0}$ can be estimated from $R_{0} \approx 1+1 / A b$, where $b$ is the per capita birth rate and $A$ the mean age at infection (8). Using country-specific data on per capita birth rates and using $A=2.3$ years from model output, the $R_{0}$ values for these six countries in 1980 were estimated to be: 9.7 (Cambodia), 10.3 (Ghana), 13.1 (India), 11.9 (Morocco), 9.6 (Nigeria), 9.4 (Uganda). Although $R_{0}=16-18$ is often used as a "rule of thumb" for the $R_{0}$ of measles, $R_{0}$ can actually vary considerably across populations; our estimate is consistent with a recent estimate of $R_{0}=8-12$ in Niamey, Niger in 2003-04 $(8,31)$.

The projected annual number of cases for the six country-level models is compared to the case reporting data in Figure 2. Despite the limited quality of the case reporting data (28), the model appears to track the trends in most of the time series from 1990 onward (but we emphasize that the model is not fitted to these case reporting data, only compared to them at baseline parameter values). The correlation coefficient $C$ between projected and observed time series are: $C=0.40$ (Cambodia), $C=0.72$ (Ghana), $C=0.66$ (India), $C=0.84$ (Morocco), $C=0.38$ (Nigeria), $C=0.02$ (Uganda). The correlation coefficient is lowest for Uganda, which has the lowest quality case reporting time series. More importantly, the model also captures the steep decline in measles cases seen in recent years: reported measles cases have been reduced virtually to zero in Cambodia, Nigeria, Uganda and Ghana due to SIAs and/or improved RI. Figure 2 also shows the projections 
of the corresponding static model, which was obtained by holding the force of infection in the dynamic model to be constant regardless of vaccination coverage. The static model does not capture the substantial decline in measles cases seen in recent years.

\section{Results}

In order to capture long-term health outcomes, we projected the total number of cases from 2008 to 2050 for all six countries except Morocco, which introduced MCV2 several years ago.

\subsection{Set \#1: Improving RI coverage}

The first set of simulations assumed improving immunization trends:

- MCV1 coverage improves by $1 \%$ or 3\% per year (additive), from 2007 onward.

- $\mathrm{MCV} 2$ is introduced at 18 months of age once MCV1 coverage reaches $70 \%$, $80 \%$ or $90 \%$.

- Follow-up SIAs are scheduled every 4, 6 or 8 years until MCV2 is introduced

MCV1 coverage was assumed to continue increasing until $90 \%$ coverage was reached

(2). MCV2 coverage was assumed to be $50 \%$ of MCV1 coverage upon introduction, then climbing by $10 \%$ of MCV1 coverage each year until it matches MCV1 coverage (2). It was assumed that the probability a child receives MCV2 does not depend on whether 
they received MCV1. For follow-up SIAs, it was assumed that every individual who did not have the opportunity to be vaccinated in the previous SIA was eligible to be vaccinated (operationally, everyone over 9 months of age at the time of the campaign but younger than the date of the most recent follow-up SIA). It was assumed that SIA coverage was $100 \%$ of eligible individuals, which reflects coverage levels reported in recent campaigns (30), though we explore the scenario of $80 \%$ coverage in sensitivity analysis. These vaccination scenarios were represented in the model by setting the vaccination rate $\phi_{\mathrm{i}}(t)$ such that the vaccine coverage described in the scenarios was recovered.

Figure 3 shows projections for Nigeria under these 18 scenarios. Both baseline and probabilistic uncertainty projections are shown. The recent lows observed in that country, relative to historical levels, are projected to continue as long as SIAs are held consistently every 4 years. However, there remains a small probability of larger numbers of cases under the probabilistic sensitivity analysis. For SIAs every 6 or 8 years, large outbreaks are probable under all scenarios except for when MCV1 coverage improves at $3 \%$ per year and MCV2 is introduced once MCV1 coverage reaches $70 \%$. Results are broadly similar for India (Supplementary Appendix 3).

Figure 4 shows the corresponding projections for Cambodia. Projected cases remain very low compared to historical levels under all scenarios. However, there are three scenarios corresponding to less optimistic improvements in RI where there is a small probability of larger numbers of cases under probabilistic sensitivity analysis (in 
particular, MCV1 improves at 1\% and MCV2 introduced at $80 \%$ MCV1 coverage; MCV1 improves at $1 \%$ and MCV2 introduced at 90\% MCV1 coverage; MCV1 improves at $3 \%$ and MCV2 introduced at $90 \%$ MCV1 coverage). For Ghana and Uganda, projected cases remain very low compared to historical norms under all scenarios, and this outcome is robust to sensitivity analysis (Supplementary Appendix 3).

Figure 2 also illustrates representative time series for the scenario: MCV1 coverage improves $1 \%$ per year; MCV2 is introduced when MCV1 coverage is $90 \%$; SIAs are held every 6 years. The time series for India and Nigeria indicate long periods of low incidence, suddenly followed by large seasonal epidemics ("honeymoon periods"). These outcomes are possible for measles in partially vaccinated populations, though within-country heterogeneity can moderate the suddenness of the effect (45). The large projected outbreaks cause the high number of cases in the projected total cases for India and Nigeria under certain scenarios.

Seasonality can be an important feature of measles dynamics (46). Hence, we investigated the impact of seasonal variation in transmission rates on model outcomes for the Nigerian scenario where MCV1 coverage improves $1 \%$ per year and MCV2 is introduced once MCV1 coverage reaches $90 \%$. In these simulations, seasonal forcing has relatively little impact on total measles cases over a 30-year time horizon, regardless of the interval between SIAs or amplitude of forcing (Supplementary Figure 1). This occurs because measles incidence over long periods of time is controlled primarily by other factors such as birth rate and population density, whereas seasonality functions primarily 
to dictate the distribution and timing of epidemic outbreaks on timescales of less than a decade (12).

\subsection{Set \#2: Stagnant RI coverage}

A second set of simulations explored scenarios where there is no improvement in MCV1 coverage and no introduction of MCV2, although SIA continues:

- Follow-up SIAs are scheduled every 2, 4, or 6 years

- Follow-up SIA coverage is $80 \%$ or $100 \%$.

Figure 5 shows the outcomes under these scenarios for Nigeria and Cambodia. For Nigeria, cases are always low when SIAs are held every 2 years, but are often high when they are held every 4 or 6 years. Results are similar for India (Supplementary Appedix 3). For Cambodia, cases are always low when SIAs are held every 2 or 4 years, but often high when they are held every 6 years. For Ghana and Uganda, cases are always low when SIAs are held every 2 or 4 years, and usually low when SIAs are held every 6 years (Supplementary Appendix 3).

\section{Discussion}

Here we have developed a model to investigate optimal second opportunity vaccination programs in low-income countries. The model was parameterized with seroprevalence 
data from low-income settings and exhibits broad agreement with data on measles incidence in low-income countries. If RI coverage continues improving, the model projects that the recent low in measles incidence (Figure 2) will continue, as long as SIAs are held at least every 4 years in India and Nigeria, and at least every 8 years in Cambodia, Ghana and Uganda. There are, however, some scenarios for India and Nigeria under which longer intervals between SIAs are possible if RI improves quickly enough. These results are robust under probabilistic sensitivity analysis, except for Cambodia where there is a small probability of large outbreaks unless SIAs are held every 4 years. If RI coverage stagnates (i.e., does not improve), then SIAs must be held more frequently to be certain of avoiding large outbreaks: every 2 years for India and Nigeria, and every 4 years in Cambodia, Ghana and Uganda.

India and Nigeria are projected to be in a "window of vulnerability" over the next 10 years, with a greater likelihood of large outbreaks until RI has improved sufficiently to stop endemic transmission. In these countries, scheduling SIAs every 2 years may be necessary to prevent large outbreaks, particularly if RI does not improve consistently. Vigilance is particularly important in these countries because of the possibility of "honeymoon periods" in recently vaccinated populations (Figure 2) (45). Both the presence of "honeymoon periods" and the dramatic difference in disease burden that occurs under a relatively minor shift of SIA interval from 4 to 6 years are a function of the nonlinear nature of measles transmission, whereby small changes in conditions can cause large changes in outcomes $(12,46)$. 
This model suggests herd immunity played an important role in achieving the virtual elimination of measles seen in some countries in recent years. For instance, the empirical and modeled time series for Morocco and Ghana suggest a very rapid decline in cases for only modest improvements in MCV1 coverage: in Morocco, the number of cases from 1980 to 1998 dropped by approximately 95\%, for an increase in MCV1 coverage to only $81 \%$. In Ghana, the number of cases from 1980 to 2000 dropped by approximately $71 \%$, while MCV1 coverage has climbed to only $73 \%$. Given a vaccine efficacy of $85 \%$ (37), this is a more rapid decline than might be implied by the eradication criterion $p_{c}=1-1 / R_{0}$, which states that $95 \%$ of individuals need to be vaccinated with a $95 \%$ efficacious vaccine to eliminate a disease with $R_{0}=10$ such as measles (8). However, this expression overestimates the proportion who must be vaccinated in cases where force of infection declines with age; this appears to hold for measles in low-income countries, though not historically in advanced countries $(8,25)$.

Current recommendations based on static models call for SIAs every 3 or 4 years, depending on the country (2). However, the optimal SIA intervals according to the present dynamic model are generally longer, and there are wider differences across countries. These differences may stem from herd immunity: as vaccine coverage level increases, the reduction in incidence due to herd immunity effects increases more rapidly, and even to the point where the disease is eliminated before $100 \%$ coverage. By comparison, in a static model, reduced incidence is linearly proportional to increased vaccination coverage. Therefore, a model that accounts for herd immunity will find that optimal SIA intervals depend more sensitively on a country's vaccination coverage, and 
that countries can often schedule SIAs less frequently than would be implied by a static model due to additional indirect protection from herd immunity. This illustrates how the type of model used in an analysis can potentially influence conclusions regarding which strategies are optimal.

We developed a relatively simple model with limited country-specific data requirements so that it could be easily adapted to any given country. However, aspects of measles epidemiology such as seasonality, stochasticity, spatial structure, social heterogeneity, and vaccine-modified measles infection can influence measles dynamics $(8,10,12,19$, $23,46-49)$. For instance, social heterogeneity may imply that vaccine coverage in some groups is lower than the national average, leading to conditions where transmission can be sustained in those subgroups even when it is not possible in the general population. Social heterogeneity is of particular concern for a country such as India, where conditions can vary substantially among federal states. This is a tradeoff in using simpler models. The model also assumed that the probability an individual receives a second dose of vaccine is independent of the probability an individual received a first dose. In reality, these probabilities are not likely to be independent. However, there appears to be little country-specific data that would allow us to estimate this probability for any given country, and when coverage for the second dose is very high (e.g., close to $100 \%$ in SIA (30)) the issue becomes less important. There is also a need for better transmission rate and vaccine coverage data with which to parameterize models, especially for older age classes that can become epidemiologically relevant as the mean age at infection rises due to rising vaccine coverage. As more data become available and dynamic modeling is 
better integrated into global health decision-making, it may be possible to develop a suite of complementary dynamic models with varying degrees of sophistication that can supplement the existing set of analytical tools for informing vaccine scheduling decisions in low-income countries.

\section{Acknowledgements}

This study was supported by the Bill \& Melinda Gates Foundation Global Health Program and the University of Washington. The authors are grateful to Colleen Burgess for comments on an earlier draft and to our program officer Girindre Beeharry and colleagues at the Bill \& Melinda Gates foundation for their support and feedback. 


\section{References}

1. Wolfson L, Strebel P, Gacic-Dobo M, et al. Has the 2005 measles mortality reduction goal been achieved? A natural history modelling study. The Lancet. 2007;369:191-200.

2. MIC. Measles Investment Case II Report; 2005.

3. Pasetti M, Resendiz-Albor A, Ramirez K, et al. Heterologous prime-boost strategy to immunize very young infants against measles: pre-clinical studies in Rhesus Macaques. Clinical pharmacology and therapeutics. 2007;82(6):672-85.

4. Cutts F, Clements C, Bennett J. Alternative routes of measles immunization: a review. Biologicals. 1997;25(3):323-38.

5. Burger J, Cape S, Braun C, et al. Stabilizing formulations for inhalable powders of live-attenuated measles virus vaccine. Journal of aerosol Medicine and Pulmonary Drug Delivery. 2008;21(1):25-34.

6. Aguado M, Henao-Restrepo A. New Measles Vaccine Formulations and Delivery Systems and their Potential Contribution to Reducing Measles Mortality Worldwide. Vaccines Preventing disease and protecting health: Pan American Health Organization; 2003. p. 43-52.

7. Hethcote H. The Mathematics of Infectious Diseases. SIAM Review. 2000;42(4):599-653.

8. Anderson R, May R. Infectious diseases of humans. Oxford: Oxford University Press; 1991.

9. Edmunds W, Medley G, Nokes D. Evaluating the cost-effectiveness of vaccination programs: a dynamic perspective. Statistics in medicine. 1999;18(23):3263-82.

10. Schenzle D. An age-structured model of pre- and post-vaccination measles transmission. IMA J Math Appl Med Biol. 1984;1:169-91.

11. Babad H, Nokes D, Gay N, Miller E, Morgan-Capner P, Anderson R. Predicting the impact of measles vaccination in England and Wales: model validation and analysis of policy options. Epidemiology and Infection. 1995;114(2):319-44. 
12. Bauch C, Earn D. Transients and attractors in epidemics. Proc Roy Soc Lond A. $2003 ; 270: 1573-8$.

13. McLean A, Anderson R. Measles in developing countries Part I. Epidemiological parameters and patterns. Epidemiology and Infection. 1988;100(1):111-33.

14. Nokes D, McLean A, Anderson R, Grabowsky M. Measles immunization strategies for countries with high transmission rates: interim guidelines predicted using a mathematical model. Int J Epidemiol. 1990;19:703-10.

15. Agur Z, Cojocaru L, Mazo G, Anderson R, 9 MG. Pulse mass measles vaccination across age cohorts. Proceedings of the National Academy of Sciences USA. 1991;90:11698-702.

16. Nokes D, Swinton J. Vaccination in pulses: a strategy for global eradication of measles and polio? . Trends in microbiology. 1997;5(1):14-9.

17. MOH. Modelling Measles: predicting and preventing measles epidemics in New Zealand. Application of a mathematical model: Ministry of Health of New Zealand; 1998.

18. Ferrari M, Grais R, Bharti N, et al. The dynamics of measles in sub-Saharan Africa. Nature. 2008;451:679-84.

19. Grenfell B, Bjornstad O, Kappey J. Travelling waves and spatial hierarchies in measles epidemics. Nature. 2001;414:716-23.

20. Hartter H, Oyedele O, Dietz K, Kreis S, Hoffman J, Muller C. Placental transfer and decay of maternally acquired antimeasles antibodies in Nigerian children. Pediatric Infectious Diseases Journal. 2000;19:635-41.

21. Janaszek W, Slusarczyk J. Immunity against measles in populations of women and infants in Poland. Vaccine. 2003;21:2948-53.

22. UN. http://esa.un.org/unpp. 2008 [cited 200824 April]; Available from:

23. Mossong J, Muller C. Modelling measles re-emergence as a result of waning immunity in vaccinated populations. Vaccine. 2003;21:4597-603.

24. Mossong J, Nokes D, Edmunds W, Cox M, Ratnam S, Muller C. Modeling the impact of sublinical measles transmission in vaccinated populations with waning immunity. American Journal of Epidemiology. 1999;150(11):1238-49. 
25. Remme J, Mandara M, Leeuwenburg J. The force of measles infection in East Africa. International Journal of Epidemiology. 1984;13(3):332-9.

26. Assaad F. Measles: summary of worldwide impact. Rev Infect Dis. 1983;5(3):452-9.

27. Perry R, Halsey N. The clinical significance of measles: a review. The Journal of Infectious Diseases. 2004;189:S4-S16.

28. WHO. http://www.who.int/immunization monitoring/data/en. 2008 [cited 2008 24 April]; Available from:

29. Hull H, Williams P, Oldfield F. Measles mortality and vaccine efficacy in rural West Africa. The Lancet. 1983;Apr 30(1(8331)):972-5.

30. MP. The Measles Partnership, http://www.measlesinitiative.org. 2008 [cited 200824 April]; Available from:

31. Grais R, Dubray C, Gerstl S, et al. Unacceptably high mortality related to measles epidemics in Niger, Nigeria and Chad. PLoS Medicine. 2007;4(1):e16.

32. Stein C, Birmingham M, Kurian M, Duclos P, Strebel P. The global burden of measles in the year 2000--a model that uses country-specific indicators. The Journal of Infectious Diseases. 2003;187(Suppl 1):S8-S14.

33. Kremer J, Schneider F, Muller C. Waning antibodies in measles and rubella vaccinees--a longitudinal study. Vaccine. 2006;24:2594-601.

34. Collier L, Oxford J. Human Virology. 3 ed: Oxford University Press; 2006.

35. AAP. Measles. In: Pickering L, editor. Red Book: 2006 Report of the Committee on Infectious Diseases. 27 ed. Elk Grove Village, IL: American Academy of Pediatrics; 2006. p. 441-52.

36. Coronado F, Musan N, Tayeb E, Haithami S, Dabbagh A, Mahoney F. Retrospective measles outbreak investigation: Sudan, 2004. Journal of Tropical Pediatrics. 2006;52(5):329-34.

37. Otten M, Okwo-Bele J, Kezaala R. Impact of alternative approaches to accelerated measles control: experience in the African region, 1996-2002. Journal of Infectious Diseases. 2003;187(Suppl 1):S36-43.

38. Dollimore N, Cutts F, Binka F, Ross D, Morris S, Smith P. Measles incidence, case fatality, and delayed mortality in children with or without vitamin A 
supplementation in rural Ghana. American Journal of Epidemiology. 1997;146(8):646-54.

39. Nandy R, Handzel T, Zaneidou M, et al. Case-fatality rate during a measles outbreak in eastern Niger in 2003. 42. 2003(322-8).

40. Samb B, Aaby P, Whittle H, Seck A, Simondon F. Protective efficacy of hightitre measles vaccines administered from the age of five months: a community study in rural Senegal. Trans R Soc Trop Med Hyg. 1993;87:697-701.

41. Lennon J, Black F. Maternally derived measles immunity in era of vaccineprotected mothers. Journal of Pediatrics. 1986;108(5):671-6.

42. Szenborn L, Tischer A, Pejcz J, Rudkowski Z, Wojcik M. Passive acquired immunity against measles in infants born to naturally infected and vaccinated mothers. Med Sci Monit. 2003;9(12):CR541-6.

43. Farrington $\mathrm{C}$, Whitaker $\mathrm{H}$. Contact surface models for infectious diseases: estimation from serologic survey data. Journal of the American Statistical Association. 2005;100(470):370-9.

44. Dabbagh A, Gacic-Dobo M, Feathersone D, Strebel P, Owko-Bele J. Progress in global measles control and mortality reduction, 2000-2006. JAMA. 2008;299(4):400-2.

45. Wallinga J, Heijne J, Kretzschmar M. A measles epidemic threshold in a highly vaccinated population. PLoS Medicine. 2005;2(11):e316.

46. Earn D, Rohani P, Bolker B, Grenfell B. A simple model for complex dynamical transitions in epidemics. Science. 2000;287:667-70.

47. Hethcote H, Ark HV. Epidemiological models for heterogeneous populations: proportionate mixing, parameter estimation and immunization programs. Mathematical Biosciences. 1987;84(11):85-118.

48. Rohani P, Earn D, Grenfell B. Opposite patterns of synchrony in sympatric disease metapopulations. Science. 1999;286:968-71.

49. Beutels P, Gay N. Economic evalution of options for measles vaccination in a hypothetical Western European country. Epidemiology and Infection. 2003;130:273-83. 


\section{Tables}

Table 1: Input model parameters.

\begin{tabular}{|c|c|c|}
\hline Parameter & Value & Reference \\
\hline Birth rate, $b(t)$ & $\begin{array}{l}\text { Varies according } \\
\text { to country, year }\end{array}$ & (22) \\
\hline Death rate by age, $d_{\mathrm{i}}(t)$ & $\begin{array}{l}\text { Varies according } \\
\text { to country, year }\end{array}$ & $(22)$ \\
\hline $\begin{array}{l}\text { Mean duration of latent } \\
\text { period, } 1 / \sigma\end{array}$ & 9 days & $(34,35)$ \\
\hline $\begin{array}{l}\text { Mean duration of effective } \\
\text { infectious period, } 1 / \gamma\end{array}$ & 5 days & $(34,35,46)$ \\
\hline $\begin{array}{l}\text { Mean rate of waning of } \\
\text { vaccine-derived immunity, } w\end{array}$ & $1.5 \%$ per year & $(24,33)$ \\
\hline Rate at which maternal & $0.39,3.8,8.3,9.1$ & Fitted; $(20,21,41,42)$ \\
\hline naturally-derived immunity & $7.7,6.0,4.5,3.2$ & \\
\hline wanes, $\rho_{i}^{N}, i=0,1,2, \ldots, 11$ & $2.3,1.6,1.1,7.3$ & \\
\hline $12+$ months & 50 per year & \\
\hline Rate at which maternal & $0.39,25.4,22.1$ & Fitted; $(20,21,41,42)$ \\
\hline vaccine-derived immunity & $2.9,12.7,19.3$ & \\
\hline wanes, $\rho_{i}^{V}, i=0,1,2, \ldots, 5$ & 55.3 per year & \\
\hline $6+$ months & & \\
\hline
\end{tabular}




\begin{tabular}{|c|c|c|}
\hline Age-specific case fatality rate & Varies according & $(2,31,32,36-39)$ \\
\hline attributable to measles, $m_{i}$ & to country & \\
\hline Vaccine coverage, $\phi_{\mathrm{i}}(t), \mathrm{RI}$ & Varies according & $(28,30)$ \\
\hline and SIA & to country, year & \\
\hline Vaccine effectiveness $\varepsilon_{\mathrm{i}}, 9-11$ & $85 \%$ & $(29,37,40)$ \\
\hline \multicolumn{3}{|l|}{ months } \\
\hline Vaccine effectiveness $\varepsilon_{\mathrm{i}},>11$ & $95 \%$ & $(29,37,40)$ \\
\hline \multicolumn{3}{|l|}{ months } \\
\hline \multirow[t]{5}{*}{ Force of infection $\lambda_{\mathrm{l}}$, per year } & $<1$ year: $40 \%$ & Fitted; see Supplementary \\
\hline & $1-4$ years: $50 \%$ & Appendix 2 for references \\
\hline & $5-9$ years: $40 \%$ & \\
\hline & $10-14$ year: $20 \%$ & \\
\hline & $15+$ years: $10 \%$ & \\
\hline
\end{tabular}




\section{Figure Captions}

Figure 1: Schematic of MSEIRV model for a single age class. Vertical grey arrows represent transfer between age classes due to aging, and curving black arrows represent deaths due to causes other than measles that are applied as individuals transfer to the next age class. Horizontal arrows represent disease processes within a single age class: arrows from $M_{\mathrm{N}, \mathrm{i}}$ and $M_{\mathrm{V}, \mathrm{i}}$ to $S_{\mathrm{i}}$ represent loss of maternal immunity; arrows from and to $S_{\mathrm{i}}$ and $V_{\mathrm{i}}$ represent vaccination and subsequent loss of vaccine-derived immunity; arrows running from $S_{\mathrm{i}}$ to $E_{\mathrm{i}}$ to $I_{\mathrm{i}}$ to $R_{\mathrm{i}}$ represent the natural disease history of infection and recovery.

Figure 2: Reported measles cases, and cases projected from dynamic and static models for 1980-2030 in Cambodia (a), Ghana (b), India (c), Morocco (d) Nigeria (e), Uganda (f). To allow comparison between reported and projected data, the under-reporting ratio was approximated as the ratio of the total number of projected cases, 1997-2006, to the total number of reported cases over the same time period. The under-reporting ratio thus computed was, for the six countries: 25.9 (Cambodia), 5.4 (Ghana), 297.5 (India), 10.1 (Morocco), 31.3 (Nigeria), and 29.5 (Uganda). The resulting under-reporting ratio was applied to the reported cases to estimate the true actual number of cases presented in Figure 1. For 2008 onward, we assumed that MCV1 coverage improves 1\% per year; MCV2 is introduced when MCV1 coverage is $90 \%$; SIAs are held every 6 years. 
Figure 3: Projected number of measles cases in Nigeria from 2008-2050 for 18 scenarios under Set \#1 (improving RI coverage). Square denotes baseline projections, and diamonds denote projections of 100 realizations under the sensitivity analysis. Note difference in vertical scale across panels.

Figure 4: Projected number of measles cases in Cambodia from 2008-2050 for 18 scenarios under Set \#1 (improving RI coverage). Square denotes baseline projections, and diamonds denote projections of 100 realizations under the sensitivity analysis. Note difference in vertical scale across panels.

Figure 5: Projected number of measles cases in Nigeria and Cambodia for 2008-2050 for 6 scenarios under Set \#1 (stagnant RI coverage), 100\% and 80\% SIA coverage. Square denotes baseline projections, and diamonds denote projections of 100 realizations under the sensitivity analysis. Note difference in vertical scale across panels. 


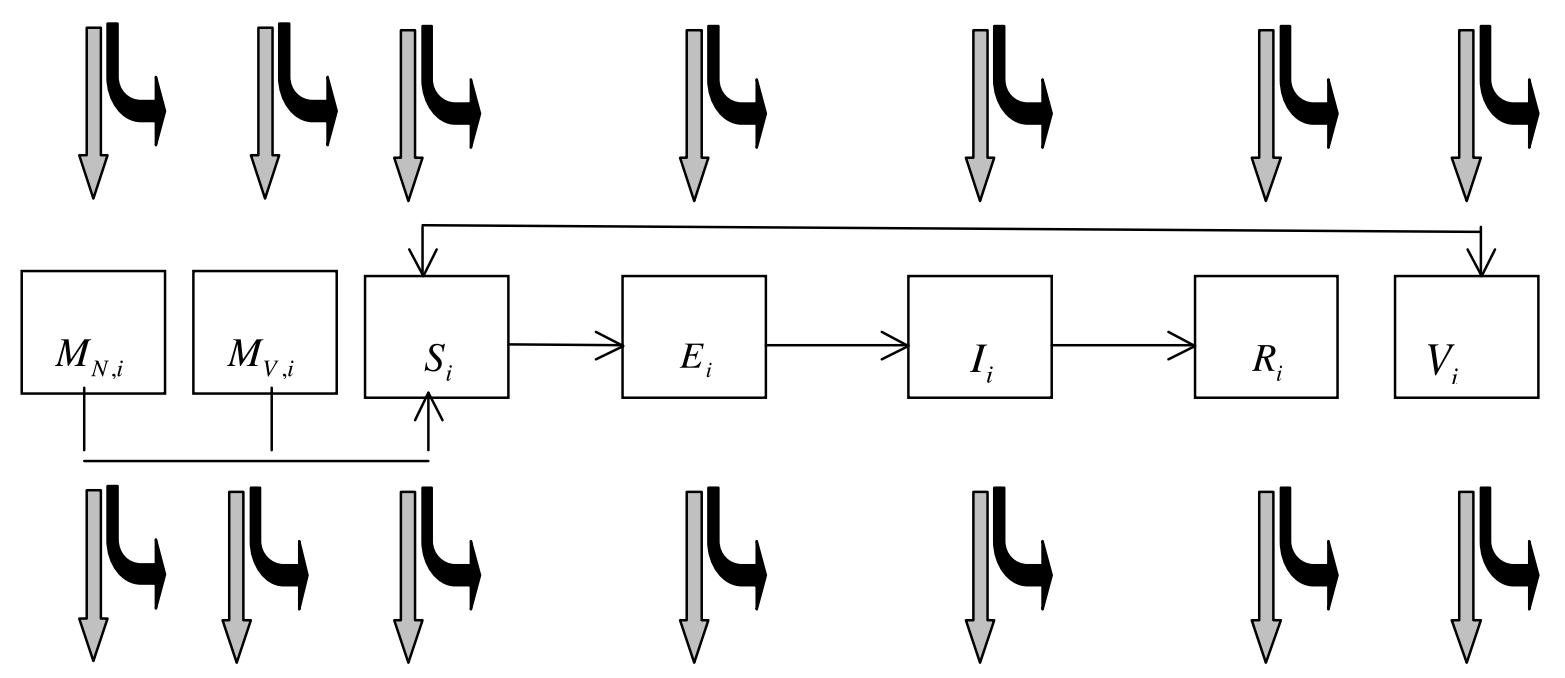



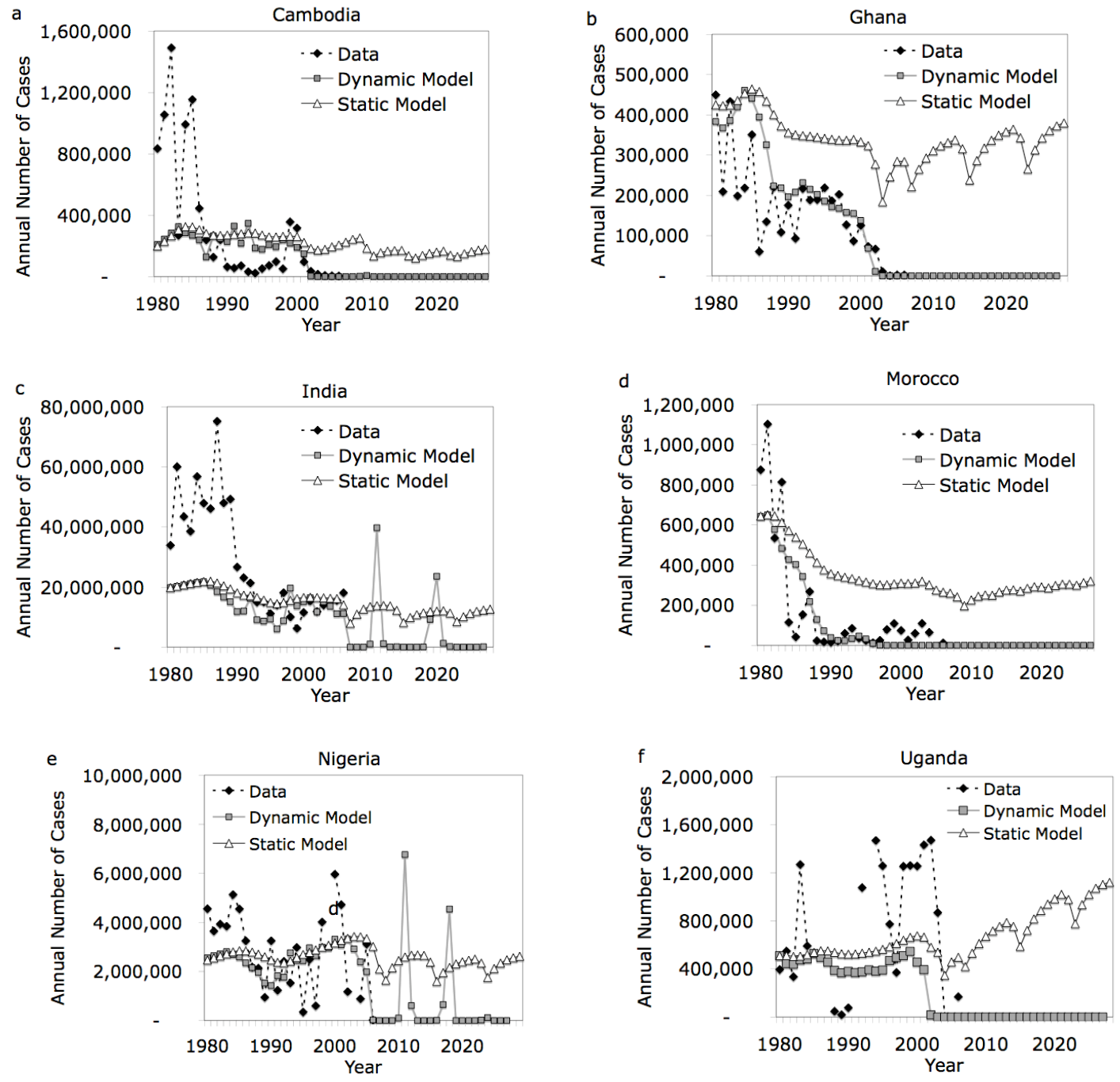

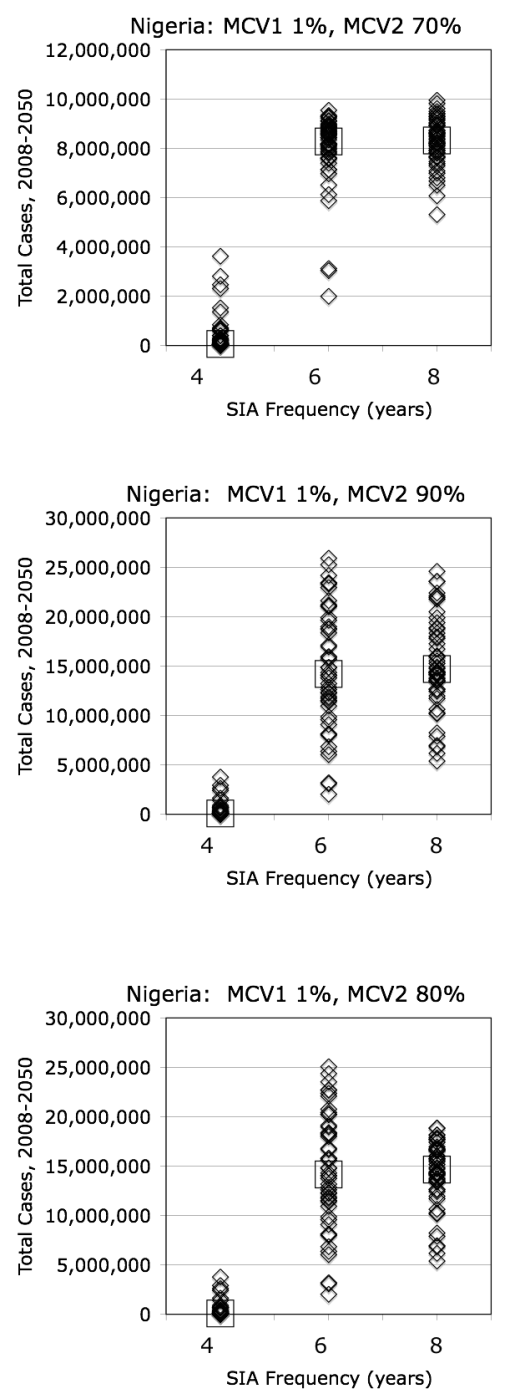
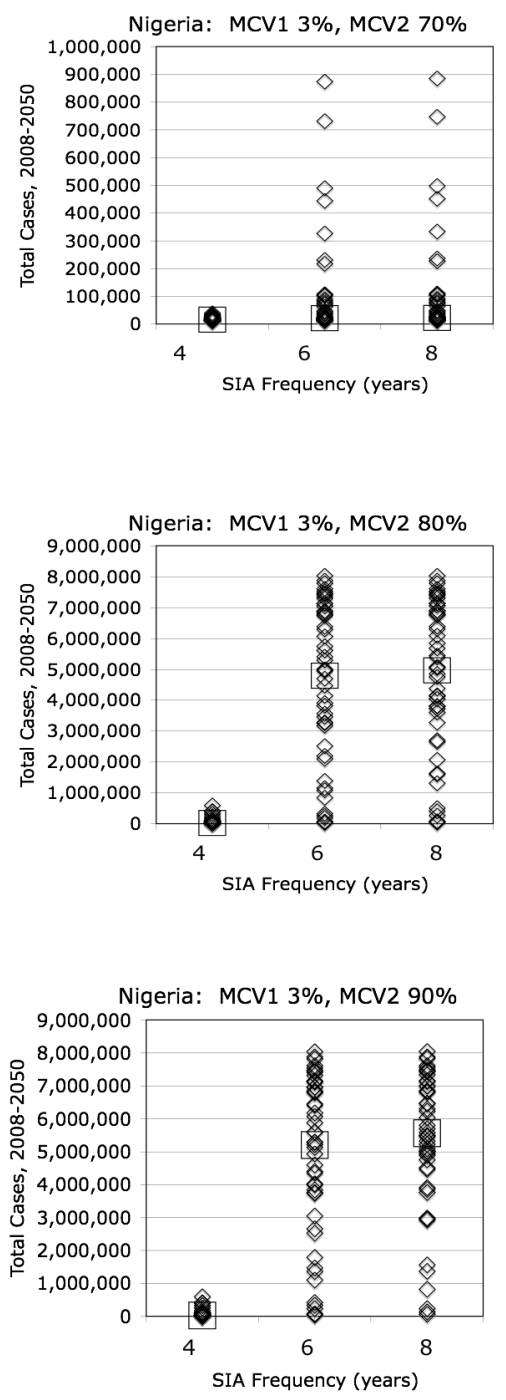

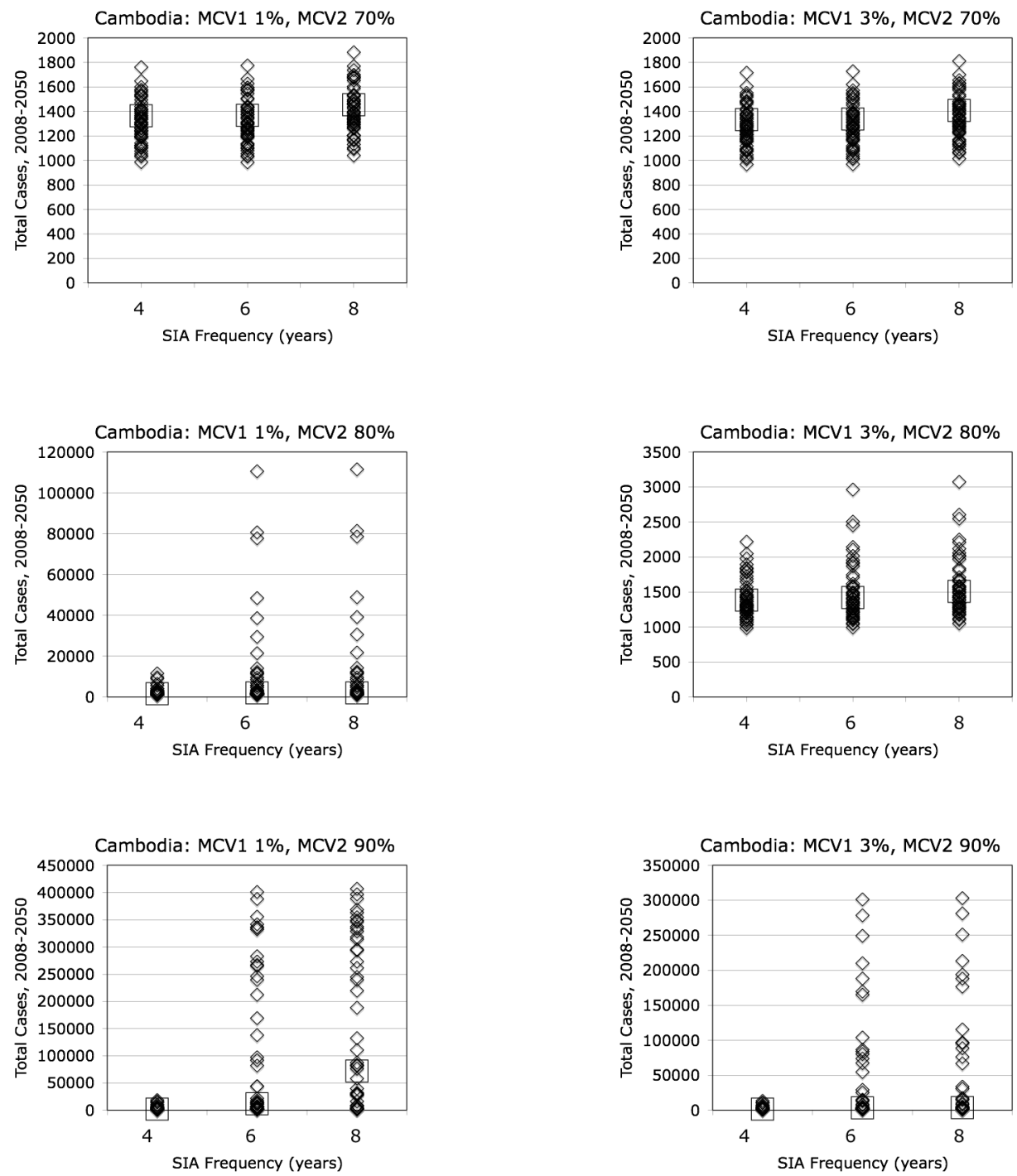

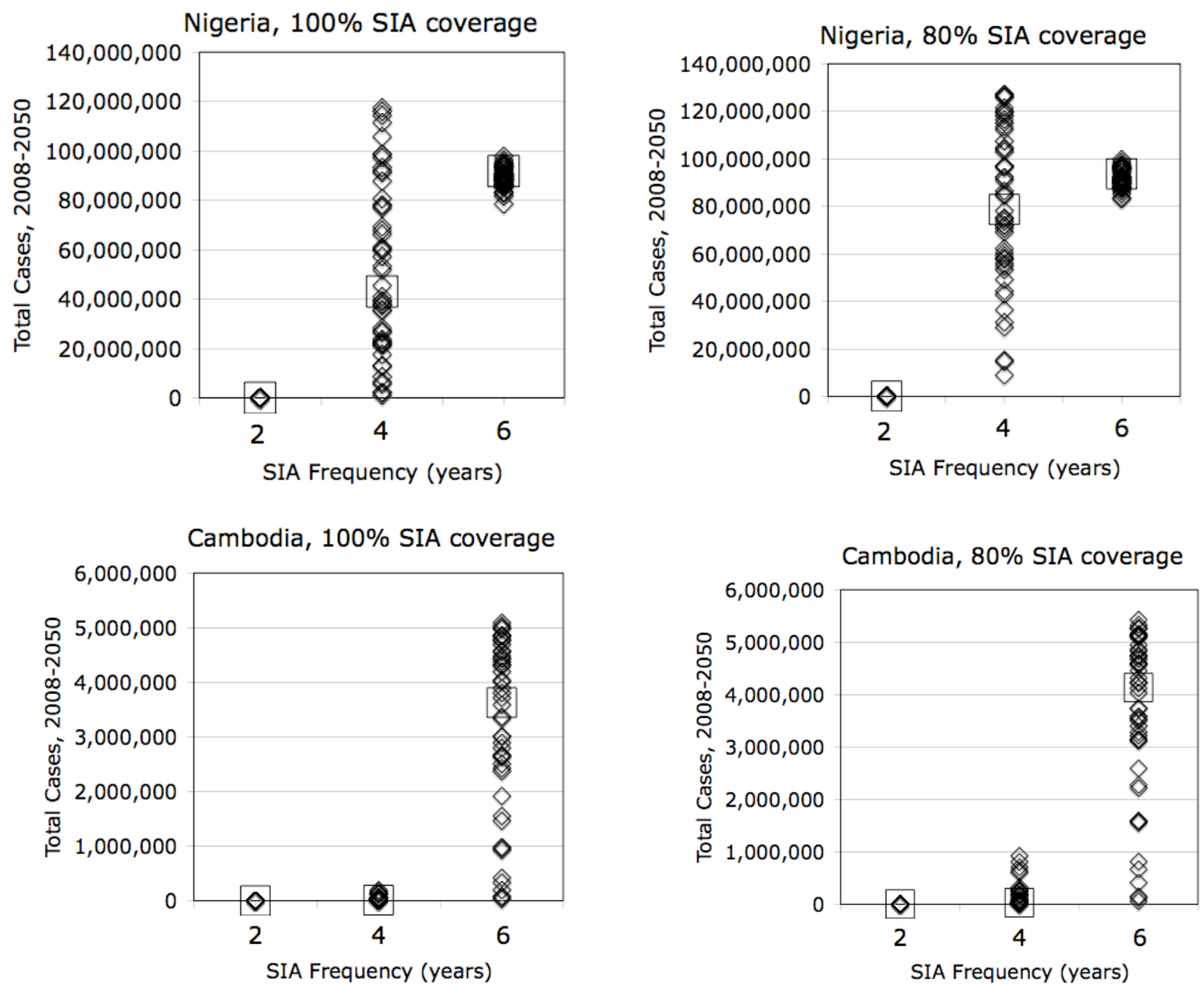